\title{
Atypical mycobacteriosis as a complication of talc pneumoconiosis
}

\author{
C. De Coster*, J.M. Verstraeten**, P. Dumortier*, P. De Vuyst*
}

Atypical mycobacteriosis as a complication of talc pneumoconiosis. C. De Coster, J.M. Verstraeten, P. Dumortier, P. De Vuyst. C CERS Journals Ltd 1996.

ABSTRACT: A 57 year old man, receiving compensation for talc pneumoconiosis since 1977, was admitted to hospital for the first time in 1987, with symptoms of weight loss, fever, dyspnoea and productive cough. A chest roentgenogram showed bilateral cavitation. Two years later, Mycobacterium xenopi was found in sputum cultures. Despite specific oral antibiotherapy, the patient's health deteriorated and he died in 1990.

To the best of our knowledge, this is the first reported case of an association of talcosis with a $M$. xenopi pneumonia. The relative timing of the two diseases suggests that talc pneumoconiosis predisposed to the infection by M. xenopi.

Eur Respir J., 1996, 9, 1757-1759.

\begin{abstract}
*Chest Dept, Erasme University Hospital, Université Libre de Bruxelles, Brussels. **Akademisch Ziekenhuis Sint Vincentius, Ghent, Belgium.
\end{abstract}

Correspondence: P. De Vuyst, Chest Service Erasme University Hospital, 808 route de Lennik, B-1070 Brussels, Belgium

Keywords: Atypical mycobacteria, talc pneumoconiosis

Received: September 191995

Accepted after revision February 201996

\section{Case report}

The patient, born in 1932, worked as a talc miller from 1969 until 1980 in a Belgian factory. The raw material was imported mainly from Montana, US (Yellowstone). Talc was crushed without addition of other materials and served as a high grade talc for pharmaceutical purposes. The patient had never previously been exposed to other minerals (e.g. silica, asbestos) and had a smoking history of 35 pack-years. In 1977, the diagnosis of talc pneumoconiosis was made, essentially on the basis of a chest radiograph. In 1983, chest radiographs showed a progression of the small opacities (graded q/q 2/2 according to

a)

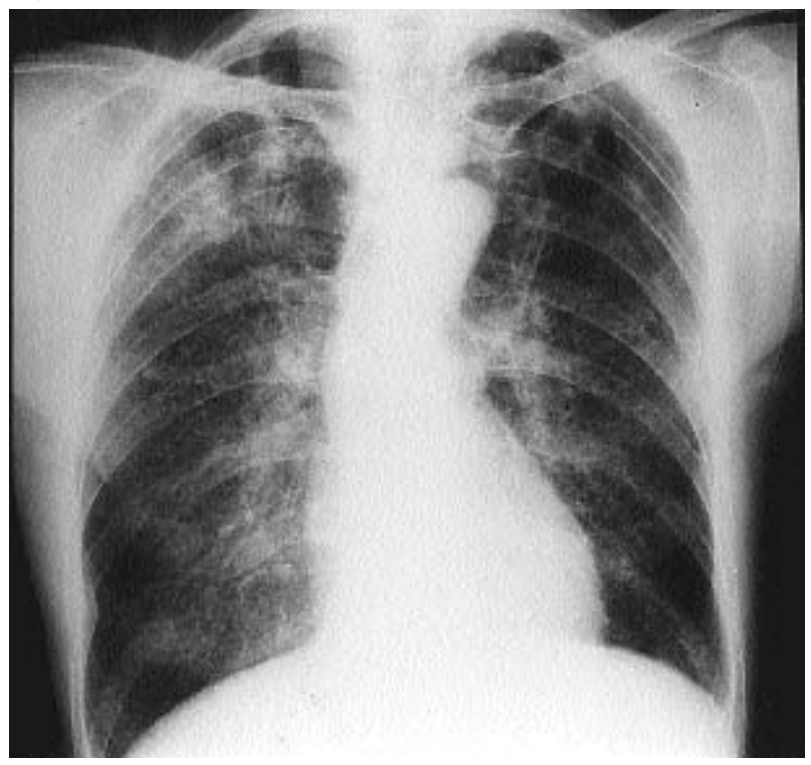

the International Labour Office (ILO) classification) and the appearance of a large opacity in the right upper lobe (graded A) (fig. 1a). The results of pulmonary function tests at that time were as follows: forced vital capacity (FVC) $2.7 \mathrm{~L}$ (65\% of predicted); forced expiratory volume (FEV1) $2.3 \mathrm{~L}$ (73\% pred); total lung capacity (TLC) $5.2 \mathrm{~L}$ (91\% pred); and transfer factor of the lung for carbon monoxide (TL,CO) $61 \%$ pred. A bronchoalveolar lavage (BAL) performed in 1983 showed abundant talc particles, talc bodies and traces of tremolite [1]. Quartz was not detected in the BAL fluid, but due to the considerable amount of talc particles, traces of quartz could have remained undetected by this method.

b)

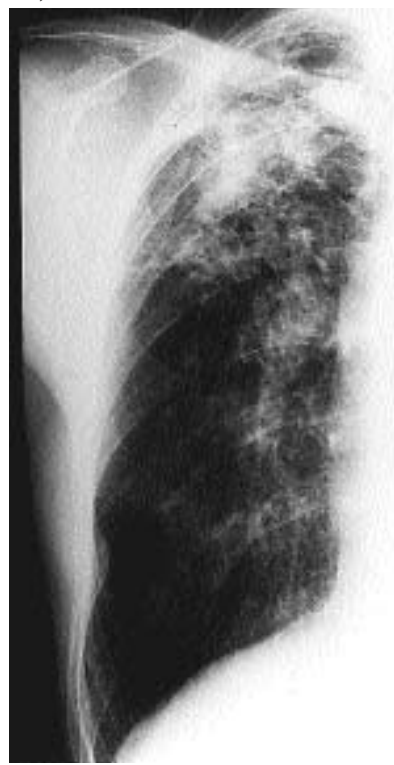

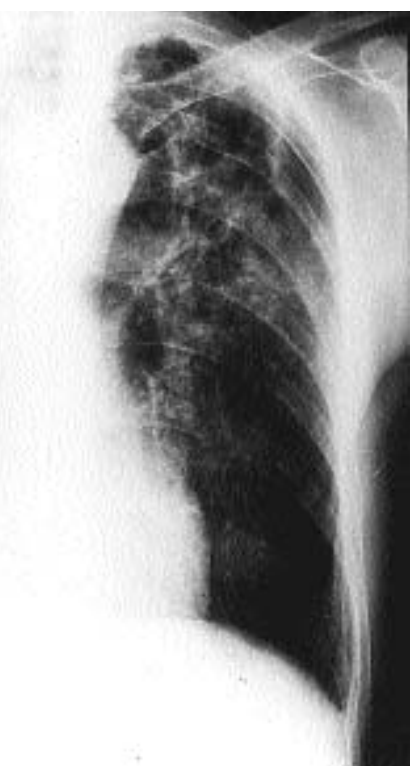

Fig. 1. - a) Chest radiograph in 1983, showing bilateral rounded opacities and a large opacity in the right upper lobe. b) Chest radiograph in 1987, showing progression of the lesions with cavitations of both upper lobes. 
In 1987, at the age of 55 yrs, the patient was admitted to the hospital with symptoms of fever, dyspnoea and purulent sputum. There was also a significant weight loss (38 kg body weight for $1.65 \mathrm{~m}$ ). Chest radiographs showed a progression of the large opacity in the right upper lobe and cavitations of both upper lobes, predominating in the left upper lobe (fig. 1b). Pulmonary function tests showed a deterioration of the spirometric values: FVC 1.4 L (35\% pred); FEV 0.6 L (21\% pred); residual volume (RV) 4.0 L (190\% pred); and TL,CO $11 \%$ pred. The sedimentation rate reached $107 \mathrm{~mm} \cdot \mathrm{h}^{-1}$ and the white blood cell count $7 \times 10^{9}$ cells $\left.\cdot \mathrm{L}^{-1}\right)$. Arterial blood gas examination disclosed severe hypoxaemia (arterial oxygen tension $\left(\mathrm{Pa}, \mathrm{O}_{2}\right)$ $4.9 \mathrm{kPa}(37 \mathrm{mmHg}))$. Sputum smears for bacteria and acid-fast bacilli were negative. Clavulanic acid + amoxycillin had no effect on the fever but the patient recovered after antibiotic treatment of 10 days duration with co-trimoxazole.

In 1988, the patient was once more admitted because of fever and chest pain. A chest radiograph showed a "hydroaeric level" in the right upper lobe. Blood cultures were negative and there were no pathogens detected in the sputum. Oral antibiotherapy with co-trimoxazole was administrated for 3 days, followed by cefuroxime. One year later, he was again admitted to the hospital with the same symptoms, chest radiographs showing a progression of the cavitary lesions.

At that time, the sputum culture yielded 20 colonies of Mycobacterium xenopi but was negative for Mycobacterium tuberculosis. Oral rifampicin, $600 \mathrm{mg} \cdot \mathrm{day}^{-1}$, and isoniazid (INH), $300 \mathrm{mg} \cdot \mathrm{day}^{-1}$, were initiated. However, because $M$. xenopi was resistant to INH, it was replaced by ofloxacin $\left(400 \mathrm{mg} \cdot \mathrm{day}^{-1}\right)$.

Four months later, the patient was admitted for the last time because his condition had deteriorated. He developed severe renal failure, anaemia, leucocytosis and shock. Despite intensive care, the patient died a few days later.

\section{Discussion}

The first period (1977-1987) of this patient's disease is dominated by the diagnosis and the compensation for talc pneumoconiosis ("occupational" period). The second is dominated by mycobacterial infection leading to death (1987-1990) ("infectious" period). Knowing the diagnosis of M.xenopi infection, it is difficult to define its exact onset. On the radiograph from 1983, it is possible that the right upper lobe opacity already corresponds to an infectious process. Nevertheless, the discussion will consider two phases in the patient's evolution: the talc pneumoconiosis and the $M$. xenopi infection, and the possible relationship between them.

Talc pneumoconiosis in an uncommon pneumoconiosis, which classically requires a long duration of exposure to a high concentration of dust $[2,3]$. Cigarette smoke appears to increase the risk of developing this disease. In the present case, the prolonged exposure to high levels of respirable talc particles, together with the presence of diffuse, bilateral, small, rounded opacities on chest radiographs is compatible with the diagnosis of talcosis $[2,3]$. BAL confirmed the retention of a very high concentration of talc particles. The clinical and radiological data were compatible with previous descriptions of pneumoconiosis in talc-exposed workers, so that no lung biopsies were taken. The Belgian Occupational Disease Fund agreed to recognize and compensate this disease.

Large opacities are also described in talcosis, and the abnormalities in the right upper lobe on the chest radiograph from 1983 were also considered as a part of the pneumoconiosis. However, the fact that this lesion was unilateral should have been considered as atypical. This underscores the problem of differential diagnosis of lung opacities appearing in pneumoconiotic subjects, usually considered as coniotic masses without any complementary investigations. Furthermore, there was a clear progression of the bilateral small opacities. No prior exposure to other mineral dust had been detected, and even if a few tremolite asbestos fibres were found in BAL, there were no evident signs of asbestosis (no pleural plaques or thickening, no evidence of interstitial fibrosis). The fact that the asbestos type was tremolite suggests a possible geological contamination of talc [1].

It is actually admitted that $M$. xenopi can be pathogenic for humans and lead to severe disease, but lung diseases due to this mycobacterial species are rare. Underlying pre-existing pulmonary diseases, such as pulmonary tuberculosis, chronic bronchitis, emphysema, bronchiectasis, interstitial pulmonary fibrosis, lung carcinoma and sarcoidosis, are predisposing factors for $M$. xenopi infection [4-6]. Diagnostic criteria exist for identifying pulmonary disease where $M$. xenopi is considered to be a pathogen, including repeated positive cultures, an abnormal chest roentgenogram consistent with mycobacterial infection, and absence of other pathogens in the sputum $[4,6]$. Various radiographic manifestations of $M$. xenopi infection are described from multiple nodular shadows to cavitary lesions [7]. The clinical and radiological evolution of our patient since 1987 is consistent with a chronic lung infection by mycobacteria. M. xenopi was found twice in sputum cultures, whereas $M$. tuberculosis was never detected and could be excluded. Therefore, according to mycobacteriological and roentgenographic criteria, it is obvious that the patient died from destructive lung infection by $M$. xenopi. There are only a few cases described in the literature in which lung disease caused by $M$. xenopi led to death $[4,5,7]$.

As far as we know, this is the first case report of the association of talc pneumoconiosis and lung infection by M. xenopi. Even if the succession of these two uncommon diseases in an individual case could be purely coincidental, this seems unlikely, and several clues suggest that the presence of talcosis predisposed to the secondary infection by M. xenopi. A parallel situation occurs in silicosis, another pneumoconiosis which is a well-known predisposing condition for tuberculosis or even nontuberculous mycobacterial disease $[8,9]$. The increased susceptibility to these other diseases could be explained by impaired function of macrophages due to silica [10, 11]. Alveolar macrophages are important in the defence against mycobacteria, not only through their phagocytic and bactericidal activities, but also through the amplification of the host defence by the release of cytokines (interleukin-1 $\beta$ (IL-1 $\beta$ ) and tumour necrosis factor- $\alpha$ $(\mathrm{TNF}-\alpha)$ ) [12]. In the case of talc pneumoconiosis, in which macrophages are overloaded by talc particles, it is conceivable that these activities can be impaired. 
In conclusion, this case study suggests that lung infection by mycobacteria, at least M. xenopi, can complicate talc pneumoconiosis. In such a situation, it should be considered as a part of the occupational disease and compensated as well.

\section{References}

1. De Vuyst P, Dumortier P, Léophonte P, Vande Weyer $\mathrm{R}$, Yernault JC. Mineralogical analysis of bronchoalveolar lavage in talc pneumoconiosis. Eur J Respir Dis 1987; 70: 150-156.

2. Léophonte P, Fabre J, Fortune JP, Pincemin J, Delaude A. Les silicatoses pulmonaires. Poumon Coeur 1978; 3: 193-201.

3. In: Parkes WE, ed. Occupational Lung Disorders. 2nd edn. London, Butterworths, 1982; Chapter 9, pp. 296-310.

4. Smith MJ, Citron KM. Clinical review of pulmonary disease caused by Mycobacterium xenopi. Thorax 1983; 38: $373-377$.
5. Banks J, Hunter AM, Campbell IA, Jenkins PA, Smith AP. Pulmonary infection with Mycobacterium xenopi: review of treatment and response. Thorax 1984; 39: 376-382.

6. Wolinsky E. Nontuberculous mycobacteria and associated diseases. Am Rev Respir Dis 1979; 119: 107-139.

7. Simor AE, Salit IE, Vellend H. The role of Mycobacterium xenopi in human disease. Am Rev Respir Dis 1987; 129: 435-438.

8. Morgan EJ. Silicosis and tuberculosis. Chest 1979; 75: 202-203.

9. Snider DE. The relationship between tuberculosis and silicosis. Am Rev Respir Dis 1978; 118: 455-460.

10. Lowrie DB. What goes wrong with the macrophage in silicosis? Eur J Respir Dis 1982; 63: 180-182.

11. Wallace RJ, O'Brien R, Glassroth J, Raleigh J, Dutt A. Diagnosis and treatment of disease caused by nontuberculous mycobacteria (ATS official statement). Am Rev Respir Dis 1990; 142: 940-953.

12. Rom NW, Zhang Y. The rising tide of tuberculosis and the human host response to Mycobacterium tuberculosis. J Lab Clin Med 1993; 121: 737-741. 